\title{
Correlación entre el ángulo de inclinación mediocarpiana y la morfología del semilunar
}

\author{
M. G. Racca ${ }^{(1)}$ L. Ardouin(1), M. García-Elías ${ }^{(2)}$ \\ INSTITUTO KAPLAN \\ (1) Licenciado En Medicina, Especialista EN Cirugía Ortopédica Y TRaumatológica, «Fellow» \\ en Cirugía De La Mano Del Instituto Kaplan \\ (2) Doctor En Medicina, Especialista En Cirugía De La Mano. Miembro Del Instituto Kaplan.
}

\author{
Correspondencia: \\ Dr. Marcelo Racca \\ Servicio de Ortopedia y Traumatología \\ Hospital de Levante \\ C/Dr Ramon y Cajal, 7 \\ 03503 Benidorm (Alicante) \\ Teléfono: (+34) 966878787 \\ Fax: 965861756 \\ e-mail: raccamarcelo@hotmail.com
}

Objetivo: Demostrar si existe correlación entre el ángulo denominado de Inclinación Mediocarpiana y el tipo de semilunar. Material y Método: Se estudiaron dos poblaciones: La primera fue de 100 pacientes con radiografías en posición póstero-anterior (PA) neutra. La segunda fue de 60 pacientes con radiografías PA en máxima desviación radial y cubital. Para cada una se determinó el tipo de semilunar por dos observadores independientes. Se propone un nuevo parámetro de análisis morfológico del carpo: el Ángulo de Inclinación Mediocarpiana.

Resultados: En la primera población, 40 casos fueron clasificados como semilunar tipo I, 47 casos como tipo II. El promedio del ángulo de inclinación mediocarpiana fue de $56,3^{\circ}( \pm 3,8)$ para el semilunar tipo I y de $67,5^{\circ}( \pm 4,2)$ para el tipo II $(\mathrm{p}<0.0001)$. En la segunda población, 23 casos fueron clasificados como semilunar tipo I, 19 casos como tipo II. El promedio de este ángulo, en las radiografías en máxima desviación radial fue de $59,5^{\circ}(\mathrm{DS} \pm 6,4)$ para el semilunar de tipo I y de $69,4^{\circ}( \pm 6)$ para el tipo II $(\mathrm{p}<0,0001)$. En las radiografías en máxima desviación cubital el promedio fue de $51^{\circ}( \pm 7,6)$ para en semilunar de tipo I y de $58,3^{\circ}( \pm 7,4)$ para el tipo II $(\mathrm{p}=0,003)$.
Objective: To demonstrate if there is a correlation between the so-called midcarpal inclination angle and the type of lunate. Material and methods: Two populations were studied: The first 100 patients with posterior-anterior (PA) radiographs in the neutral position. The second 60 patients with radiographs in full radial and ulnar deviation. In each group, two independant observers assessed the type of lunate. We propose a new parameter for the morphological analysis of the carpus: the midcarpal inclination angle.

Results: In the first population, 40 cases were classified as lunate type I, 47 cases as type II. The average midcarpal inclination angle was $56.3^{\circ}( \pm 3.8)$ for the lunate type I and $67.5^{\circ}( \pm 4.2)$ for type II (p $<0.0001)$. In the second population, 23 cases were classified as lunate type I, 19 cases as type II. The average of this angle, in full radial deviation on the radiographs were $59.5^{\circ}(\mathrm{SD} \pm 6.4)$ for the lunate type I and $69.4^{\circ}( \pm 6)$ for type II $(\mathrm{p}<0.0001)$. On the full ulnar deviation radiographs, the average was $51^{\circ}( \pm 7.6)$ for the lunate type I and $58.3^{\circ}( \pm 7.4)$ for the type II $(\mathrm{p}=0.003)$. Conclusions: This new midcarpal inclination angle offers a tool to determine the type of lunate radiologically, independently 
Conclusiones: Este nuevo ángulo de inclinación mediocarpiana ofrece una herramienta más para determinar radiológicamente el tipo de semilunar, independientemente de la posición de la muñeca en el momento de obtener la radiografía.

Palabras Clave: semilunar, morfología, muñeca, ángulo. of the position of the wrist at the time of obtaining the radiograph.

Keywords: lunate, morphology, wrist, angle.

\section{INTRODUCCIÓN}

$\square$ esde la publicación del Tratado de Anatomía Humana de Testut $^{1}$, se sabe que el semilunar puede tener una faceta articular medial destinada al polo proximal del ganchoso. En su tesis doctoral de 1966, Antuña Zapico $^{2}$ describió la morfología del semilunar estudiando 50 piezas cadavéricas, encontrando una faceta articular medial del semilunar en un $24 \%$ de los casos.

En publicaciones posteriores ${ }^{3-5}$, este hecho ha sido ocasionalmente mencionado hasta que en 1990 Viegas et al. ${ }^{6}$ publicaran la incidencia, la variación, las condiciones patológicas asociadas y la posible importancia clínica de la faceta medial del semilunar en la disección de 165 muñecas cadavéricas. Según este estudio se clasifica al Semilunar en tipo I (una sola faceta articular distal) y tipo II (dos facetas articulares distales). En ese mismo estudio y en publicaciones posteriores $^{7,8}$ se afirma que facetas mediales muy pequeñas pueden no ser vistas en radiografías planas.

Posteriormente, Nakamura et al. ${ }^{9}$ demostraron que la distancia entre el piramidal y el hueso grande en radiografías Póstero-Anteriores (PA) en posición neutra, aumenta en relación a la existencia de una faceta medial del semilunar. Así propuso identificar al semilunar tipo I y II según la distancia entre piramidal-hueso grande.

En este trabajo se presenta una opción alternativa según la cual puede deducirse la tipología del semilunar en el momento de obtener la radiografía sea cual sea la posición de la muñeca. El objetivo de este estudio es demostrar que existe una correlación estadísticamente significativa entre el ángulo denominado de inclinación mediocarpiana y el tipo de semilunar.

\section{MATERIAL Y MÉTODO}

Se estudiaron dos poblaciones de pacientes:

La primera población se obtuvo de la revisión de las historias clínicas de 100 pacientes con radiografías normales de muñeca en posición PA neutra según técnica estándar (hombro en abducción de $90^{\circ}$ y codo en flexión de $90^{\circ}$ ). La edad promedio fue de 46 años ( \pm 17$)$.

La segunda población estudiada consiste en 60 voluntarios normales (30 hombres y $30 \mathrm{mu}-$ jeres), sin antecedentes de traumatismos previos de la muñeca, con un promedio de edad de 32,4 años $( \pm 5,4)$. Dos radiografías en proyección PA fueron obtenidas para cada individuo según técnica estándar. La primera fue hecha en máxima desviación radial y la segunda en máxima desviación cubital.

Para cada población, se determino el tipo de semilunar, en donde dos investigadores independientes (MR y LA) clasificaron al semilunar en tipo I y tipo II por observación directa. Solo fueron incluidos para esta investigación los casos en donde hubo plena coincidencia en la clasificación. Los casos en donde no hubo coincidencia quedaron excluidos de este estudio.

Se propone un nuevo parámetro de análisis morfológico del carpo: el Ángulo de inclinación mediocarpiana. Para medir este ángulo, se traza 
Tabla I - Resultados de la población con radiografías en proyección PA Neutra.

\begin{tabular}{|l|c|c|c|}
\cline { 2 - 4 } \multicolumn{1}{c|}{} & Semilunar Tipo I & Semilunar Tipo II & (p) \\
\hline Número (\%) & $40(40 \%)$ & $47(47 \%)$ & \\
\hline${ }^{\circ}$ Prom. Proyección Neutra (DE) & $53,3^{\circ}( \pm 3,8)$ & $67,5^{\circ}( \pm 4,2)$ & $<0,0001$ \\
\hline
\end{tabular}

(\%) Porcentaje del total de la muestra

(DE) Desviación Estándar

(p) Valor de significación estadística $<0,05$

una línea que une tres puntos bisectrices de la diáfisis del tercer metacarpiano. Esta línea representa el eje del metacarpiano. Otra línea corresponde a la tangente de la parte más convexa y proximal del hueso grande y del ganchoso. Este ángulo fue medido en las radiografías de la muñeca en posición neutra (Figura 1), y con la muñeca en desviación radial y cubital (Figura 2)

Para determinar la reproductibilidad de estas mediciones, tres observadores independientes, midieron el ángulo de inclinación mediocarpiana en 15 radiografías PA de muñeca en posición neutra, desviación radial y cubital, tres veces en días consecutivos. Se cuantificó el error de medición con un coeficiente de correlación interobservador e intra-observador.

Análisis estadístico: Las características basales de las cohortes evaluadas fueron reportadas en porcentajes para los datos categóricos y en promedio con su desviación estándar (DE) para los datos continuos. Para el análisis estadístico de las muestras no apareadas fue utilizado el Test de la T de Student («two-sample test»). Valores de $\mathrm{p}<0,05$ fueron considerados significativos. Para evaluar el error de medición inter-observador e intra-observador se utilizó el coeficiente
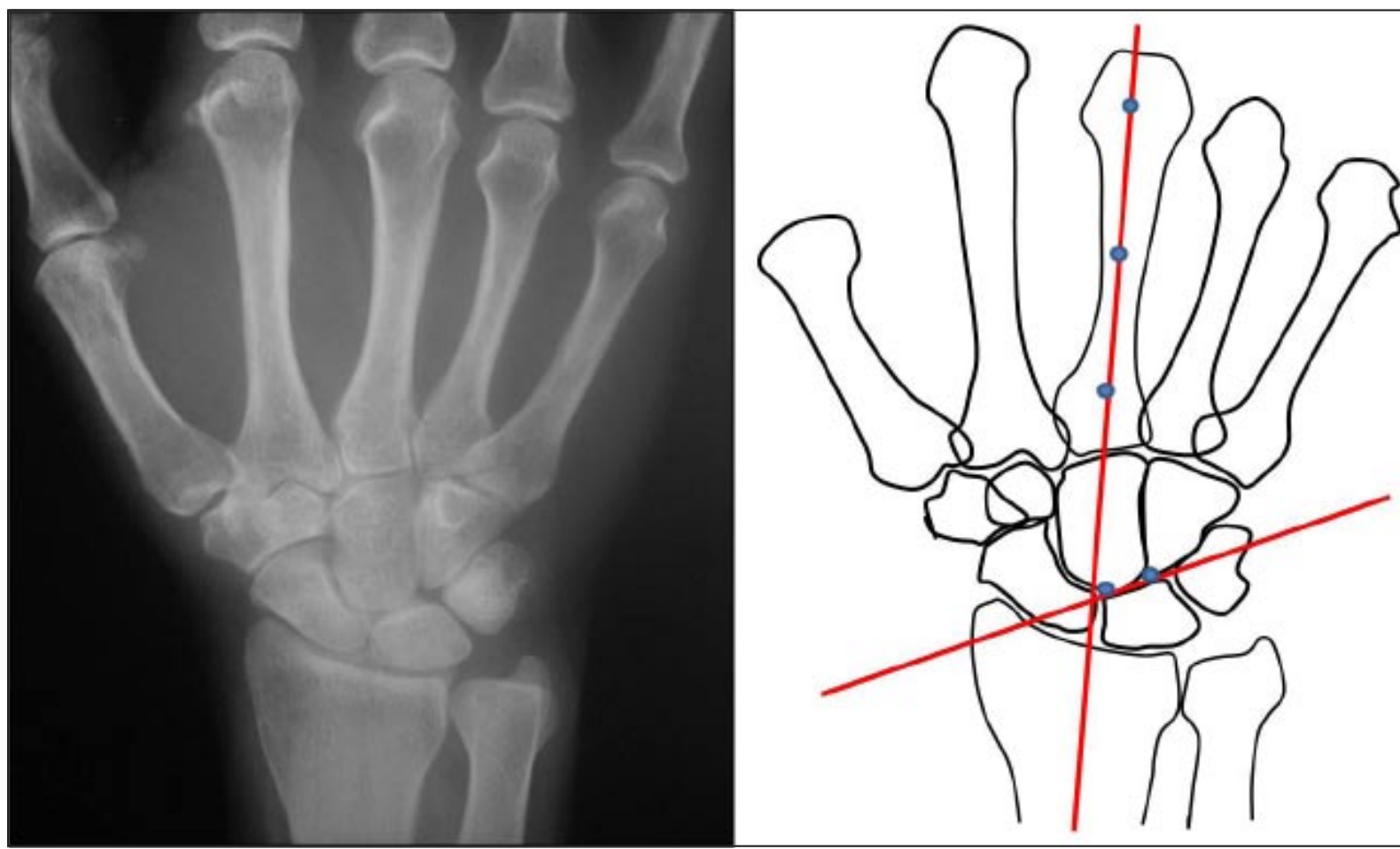

Figura 1: Medición del ángulo de inclinación Mediocarpiana en radiografías PA de muñeca en posición Neutra. 
Tabla II - Resultados de la población con Radiografías en desviación Radial y Cubital.

\begin{tabular}{|l|c|c|c|}
\cline { 2 - 4 } \multicolumn{1}{c|}{} & Semilunar Tipo I & Semilunar Tipo II & (p) \\
\hline Número (\%) & $23(38 \%)$ & $19(32 \%)$ & $<0,0001$ \\
\hline${ }^{\circ}$ Prom. Desv Radial (DE) & $59,5^{\circ}( \pm 6,4)$ & $69,4^{\circ}( \pm 6)$ & 0,003 \\
\hline${ }^{\circ}$ Prom. Desv Cubital (DE) & $51^{\circ}( \pm 7,6)$ & $58,3^{\circ}( \pm 8,4)$ & \\
\hline
\end{tabular}

(\%) Porcentaje del total de la muestra

(DE) Desviación Estándar

(p) Valor de significación estadística $<0,05$

de correlación de Pearson. La recolección y el análisis de los datos se realizaron con el programa Minitab 16 Statistical software.

\section{RESULTADOS}

En la primera población estudiada, se estableció que en 87 de 100 casos (87\%) hubo plena coincidencia entre los observadores en el tipo de semilunar. Del total, 40 casos $(40 \%)$ fueron clasificados como semilunar tipo I, 47 casos (47\%) como tipo II y 13 casos (13\%) fueron indeterminados.

Para la segunda población, se encontró que en 42 de los 60 pares radiológicos (70\%), hubo plena coincidencia en el tipo de semilunar. Veintitrés casos (38\%) fueron clasificados como semilunar tipo I, 19 casos (32\%) como semilunar tipo II y 18 casos (30\%) fueron clasificados como semilunares indeterminados.

El promedio del ángulo de inclinación mediocarpiana en las radiografías en posición PA neutra de la muñeca para el semilunar tipo I fue de $56,3^{\circ}(\mathrm{DE} \pm 3,8)$ y para el semilunar de tipo II fue de $67,5^{\circ}(\mathrm{DE} \pm 4,2)$, relación que resulto ser estadísticamente significativa $(\mathrm{p}<0.0001)$.

\section{(Tabla I)}

El promedio del ángulo de inclinación mediocarpiana en la proyección PA en máxima desviación radial fue de $59,5^{\circ}(\mathrm{DE} \pm 6.4)$ para el semilunar de tipo I y de $69,4^{\circ}(\mathrm{DE} \pm 6)$ para el semilunar de tipo II. El promedio de este mismo ángulo para el semilunar de tipo I en máxima desviación cubital fue de $51^{\circ}(\mathrm{DE} \pm 7,6)$ y de $58,3^{\circ}(\mathrm{DE} \pm 7.4)$ para el semilunar tipo II. En
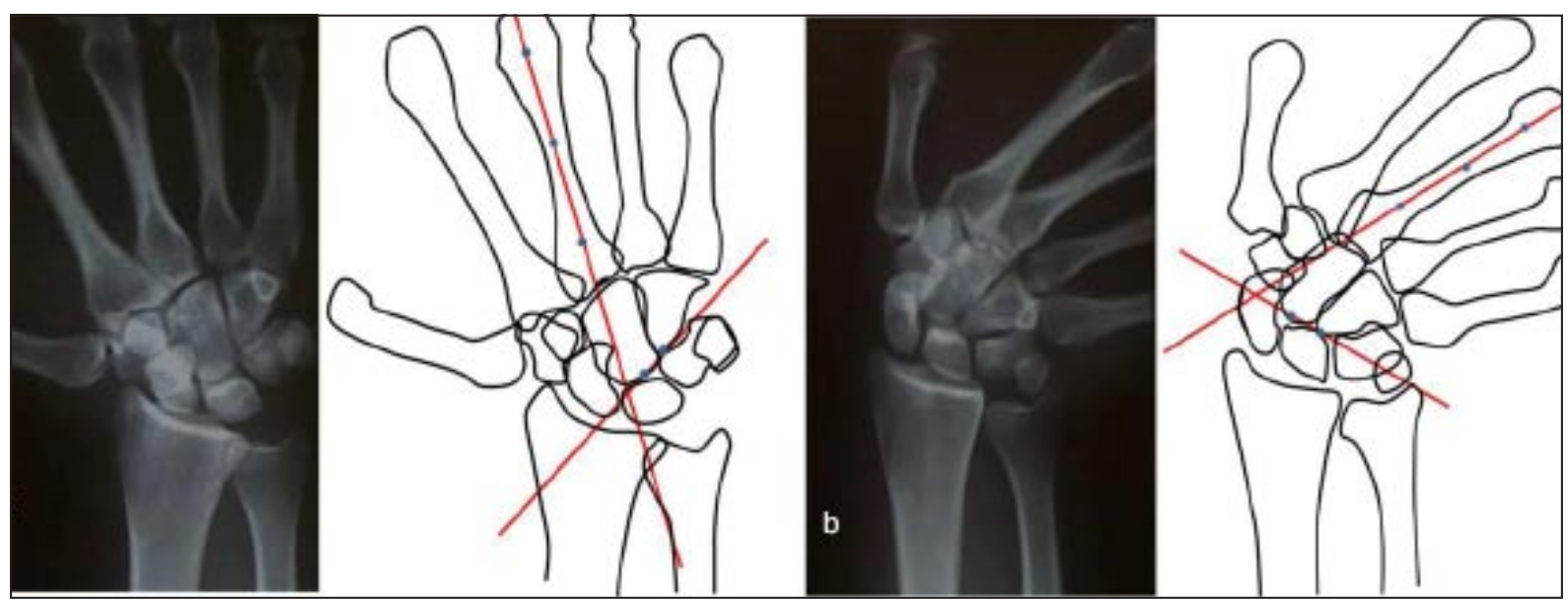

Figura 2: Medición del ángulo de inclinación Mediocarpiana en radiografías PA de muñeca en máxima desviación Radial (a) y máxima desviación Cubital (b). 
ambos casos la diferencia resulto ser estadísticamente significativa $(\mathrm{p}<0,0001 \mathrm{y} p=0,003$ respectivamente). (Tabla II)

El coeficiente de correlación de Pearson, utilizado para cuantificar los errores de medición inter-observador e intra-observador, fue de 0.86 y 0.84 respectivamente.

\section{DISCUSIÓN}

La incidencia de una faceta articular medial del semilunar para el ganchoso ha sido, según Antuña Zapico ${ }^{2}$, de 12 sobre 50 especímenes de semilunares (24\%), obtenidos de la osteoteca de la Universidad de Valladolid. En 19 de 50 (38\%) no hubo faceta articular; y en los otros 19 especímenes (38\%), no se pudo determinar si había o no faceta articular medial del semilunar. Estos resultados son comparables a los que hemos evidenciados en el presente estudio.

Viegas et al. ${ }^{6}$ identificaron un $34,5 \%$ de semilunares Tipo I en la disección de 165 muñecas cadavéricas y $65,5 \%$ de semilunar tipo II, en donde la faceta medial media de 1 a $6 \mathrm{~mm}$ de ancho. En el examen radiográfico, encontró que el semilunar tipo I fue evidente en el 46,1\% de los casos y el semilunar tipo II fue evidente en el 53\%. El porcentaje ligeramente superior del semilunar tipo I encontrado en este estudio, con respecto a la anatómica, puede deberse a que facetas articulares mediales muy pequeñas no siempre pueden evidenciarse en la radiografías simples.

Posteriormente Nakamura et $\mathrm{al}^{8,9}$ llegan a la misma conclusión sobre la dificultad en determinar el tipo de semilunar sobre radiografías planas; y proponen medir la distancia entre el Piramidal (Pir) y el Hueso Grande (HG) en radiografías de frente en posición neutra, como método para identificar al tipo de semilunar. Así sostuvo que distancias iguales o menores a $2 \mathrm{~mm}$ corresponden a un semilunar Tipo I; distancias iguales o mayores a $4 \mathrm{~mm}$ al semilunar tipo II; y que las distancias entre 2,1 $\mathrm{mm}$ y $3,9 \mathrm{~mm}$ corresponden a un tipo de semilunar no determinado. El inconveniente que este método ofrece para determinar el tipo de semilunar, es que las radiografías deben ser realizadas estrictamente en posición neutra, ya que pequeñas desviaciones hacia cubital o radial, modifica esta distancia.
La ventaja que aporta la medición del ángulo de inclinación mediocarpiana en el diagnóstico de la morfología del semilunar es que puede ser utilizado con la muñeca en cualquier posición. Con los resultados obtenidos, un ángulo de inclinación mediocarpiana inferior a $60^{\circ}$ tiene altísimas probabilidades de que el semilunar sea de tipo I y un ángulo superior a $60^{\circ}$ a un semilunar de tipo II. Los resultados obtenidos en el coeficiente de correlación de Pearson, utilizado para evaluar el grado de error inter e intra-observador, avalan la reproductibilidad del método.

Existen diversos motivos por los cuales es interesante conocer si el semilunar tiene una o dos carillas articulares distales. Por una parte, podemos estimar el comportamiento cinemático de la primera fila del carpo. Galley et al. ${ }^{10}$ publicaron la influencia del tipo de semilunar sobre la cinemática del escafoides, y encontraron que para los semilunares de tipo II el escafoides se comporta según un patrón columnar de Craigen y Stanley ${ }^{11,12}$ mientras que el tipo I, lo hace según un patrón de fila.

También, conociendo la morfología del semilunar, se podría prever el comportamiento del semilunar bajo ciertas condiciones patológicas. Haase et al. ${ }^{13}$, publicaron que en las seudoartrosis de escafoides, los semilunares tipo II, tienen una baja incidencia de inestabilidad del segmento intercalar dorsal (DISI), en comparación con semilunares tipo I. Es más, los semilunares tipo II suelen producir lesiones por impactación ganchoso-semilunar (condromalacia polar proximal del ganchoso) mientras que el semilunar tipo I nunca tendrá este problema.

En conclusión, creemos que la introducción de este nuevo ángulo de Inclinación Mediocarpiana ofrece una herramienta más para determinar radiológicamente el tipo de semilunar, con la ventaja de que, en comparación con el parámetro Pir-HG descripto por Nakamura et al. ${ }^{8}$, éste puede ser medido sea cual sea la posición de la muñeca en el momento de obtener la radiografía.

\section{TRATAMIENTO ESTADÍSTICO}

Las características basales de las cohortes evaluadas fueron reportadas en porcentajes para los datos categóricos y en promedio con su des- 
viación estándar (DE) para los datos continuos. Para el análisis estadístico de las muestras no apareadas fue utilizado el Test de la T de Student («two-sample test»). Valores de $\mathrm{p}<0,05$ fueron considerados significativos. Para evaluar el error de medición inter-observador e intra-observador se utilizó el coeficiente de correlación de Pearson. La recolección y el análisis de los datos se realizaron con el programa Minitab 16 Statistical software.

\section{BIBLIOGRAFÍA}

1. Testut L. Tratado de Anatomía Humana. $7^{\text {a }}$ edición. Vol. I. Barcelona: Editorial Salvat, 1921: 343.

2. Antuña Zapico JM. Morfología radiológica de los huesos del carpo. En: Antuña Zapico JM. Malacia del Semilunar. Tesis Doctoral. Universidad de Valladolid. Secretariado de Publicaciones. 1966: 85-148.

3. Bogumill GP. Anatomy of the wrist. En: Lichtman DM (Ed). The Wrist and its Disorders. Philadelphia: WB Saunders, 1988: 16.

4. Steele DG, Bramblett CA. The Anatomy and Biology of the Human Skeleton. Texas A\&M University Press, College Station: 1988: 176-7.

5. Kauer JMG, Landsmeer JMF. Le poignet. En: Tubiana $R(E d)$.
Traité de Chirurgie de la Main. Paris: Masson, 1980: 176-91.

6. Viegas SF, Wagner K, Patterson R, Peterson P. Medial (hamate) facet of the lunate. J Hand Surg Am, 1990; 15: 564-71.

7. Viegas SF, Patterson RM, Hokanson JA, Davis J. Wrist anatomy: incidence, distribution, and correlation of anatomic variations, tears, and arthrosis. J Hand Surg Am, 1993; 18: 463-75.

8. Nakamura K, Beppu M, Patterson RM, Hanson CA, Hume PJ, Viegas SF. Motion analysis in two dimensions of radialulnar deviation of type I versus type II lunates. J Hand Surg Am, 2000; 25: 877-88.

9. Nakamura K, Beppu M, Matushita K, Arai T, Ide T. Biomechanical analysis of the stress force on midcarpal joint in Kienbock's disease. Hand Surg, 1997; 2: 101-15.

10. Galley I, Bain G, McLean, JM. Influence of lunate type on scaphoid kinematics. J Hand Surg Am, 2007; 32: 842-7.

11. Craigen MA, Stanley JK. Wrist kinematics. Row, column or both? J Hand Surg Br, 1995; 20: 165-70.

12. García-Elías M, Ribé M, Rodríguez J, Cots $M$, Casas $J$ Influence of joint laxity on scaphoid kinematics. J Hand Surg $\mathrm{Br}, 1995 ;$ 20: 379-82.

13. Haase SC, Berger RA, Shin AY. Association between lunate morphology and carpal collapse patterns in scaphoid nounions. J Hand Surg Am, 2007; 32: 1009-12. 